Asian J. Med. Biol. Res. 2020, 6 (1), 123-129; doi: 10.3329/ajmbr.v6i1.46486

\author{
Asian Journal of \\ Medical and Biological Research \\ ISSN 2411-4472 (Print) 2412-5571 (Online) \\ www.ebupress.com/journal/ajmbr
}

\title{
Article \\ Survival probabilities of stomach and colon cancer patients in Bangladesh
}

Tanvir Ahammed ${ }^{1}$, Mst. Farzana Akter ${ }^{1}$, Afroza Jannat Suchana ${ }^{1}$, Anisa Ahmed Chowdhury ${ }^{1}$, Md. Rafil Tazir Shah $^{1}$, Md. Siddkur Rahman ${ }^{2}$, Ayesha Akter ${ }^{3}$ and Mohammad Ohid Ullah ${ }^{1 *}$

${ }^{1}$ Department of Statistics, Shahjalal University of Science and Technology, Sylhet 3114, Bangladesh

${ }^{2}$ Department of Statistics, Begum Rokeya University, Rangpur, Bangladesh

${ }^{3}$ Department of Statistics, M. C. College, Sylhet, National University, Bangladesh

*Corresponding author: Mohammad Ohid Ullah, Department of Statistics, Shahjalal University of Science and Technology, Sylhet 3114, Bangladesh. E-mail: ohid-sta@sust.edu

Received: 29 February 2020/Accepted: 22 March 2020/ Published: 31 March 2020

\begin{abstract}
Cancer is predicted to be a progressively vital reason behind morbidity and mortality in the next few decades, in all regions of the world. In Bangladesh, about 150781 new cancer cases occur in 2018 and caused about 108137 deaths. Stomach and colon cancer have $6.3 \%$ and $2 \%$ of the total cancer deaths in Bangladesh. However, to the best of knowledge, no study has been conducted in Bangladesh on the survival probabilities of stomach and colon cancer patients. Therefore, we aimed to conduct a study on the survival probabilities of stomach and colon cancer patients with respect to sex, age, and body mass index. Only sixty-five patients with stomach or colon cancer from the eight divisional cities in Bangladesh were observed. A non-parametric, Kaplan-Meier product limit estimate for survival probabilities was utilized in this study. Less median survival time for female patients (22 months) than for males (31 months) was found. Almost similar patterns were observed for patients older than 45 years compared to younger patients and overweight patients. Taken together, this study suggests that male, younger and healthy weight patients' survival probabilities are higher than female, older and overweight patients respectively. To avoid these kinds of diseases and increase survival probabilities, we should always take a balanced diet and doing exercise regularly to keep a healthy weight. Government and non-government organizations need to take proper steps to create awareness levels among the people as well.
\end{abstract}

Keywords: cancer; stomach; colon; K-M estimates; survival probabilities

\section{Introduction}

Cancer, additionally referred to as malignancy, is an abnormal growth of cells. Cancer is the leading reason for death in economically developed countries (World Health Organization, 2008) and the second leading reason behind death in developing countries and is predicted to be a more and more important reason behind morbidity and mortality within the next few decades, in all regions of the world. In economically developing countries the burden of cancer is increasing as a result of population aging and growth likewise as, increasingly, an adoption of cancer-associated lifestyle choices including smoking, physical inactivity, and "westernized" diets (Jemal et al., 2011). In Bangladesh, about 150781 new cancer cases occur in 2018 and caused about 108137 deaths.

There are more than hundred types of cancer, including breast cancer, skin cancer, lung cancer, stomach cancer, colon cancer, prostate cancer, and lymphoma. Around the world, lung cancer is the most commonly diagnosed cancer ( $11.6 \%$ of the total cases) and the leading reason behind cancer death (18.4\% of the total cancer deaths), followed by colorectal cancer (9.2\%), stomach cancer (8.2\%), and liver cancer (8.2\%) (Jemal et al., 2011) wherein Bangladesh stomach and colon cancer have $6.3 \%$ and $2 \%$ of the total cancer deaths with incidence rates of $4.8 \%$ and $1.8 \%$ respectively (Bray, 2018). In Europe during 2000, the incidence for all cancers combined ranged from 400 cases per 100,000 for age group 50-54 to 2280 for age group 70-74 in men and from 490 to 1210 in women. Over $65 \%$ of deaths from cancer occurred in elderly patients aged 65 years or more (Ferlay et al., 2004). A very large difference in prognosis also has been observed between the elderly and younger patients 
(Janssen-Heijnen et al., 2005). In colon cancer diagnosing age is also reciprocally associated with prognosis (Sant et al., 1995).

Some population-based studies on cancer patients support the concept of the role of gender in predicting survival. It appears male patients were more susceptible to die from colon cancer. This finding is confirmed by some studies (Capocaccia et al., 1997; Li et al., 2007) however, there are some controversies (Cheng et al., 2001; Ji et al.,1998; Svensson et al., 2002) as well.

A stronger association between body mass index and colon and/or rectal cancer in men than in women have additionally been delineated in numerous studies (Wynder et al., 1967; Lew et al., 1979; Phillips et al., 1985; Wu et al., 1987; Graham et al., 1988; Kune et al., 1990). Among women, the risk is modified by menopause status, possibly through altered endogenous estrogen levels (Hou et al., 2006). It has been hypothesized that feminine sex steroids offer women protection both from the disease and in terms of survival (Hayne et al., 2001; Payne, 2007).

Stomach cancer is a major health problem in many countries. Stomach cancer is a vital contributor to the worldwide burden of cancer (Van Cutsem et al., 2016) and less than a century ago it was the most common cancer in the world (Karimi et al., 2014). Stomach cancer, or gastric cancer, is a build-up abnormal cells that form a mass in part of the stomach. It can develop in any part of the body. According to the WHO, stomach cancer caused 783,000 deaths worldwide in 2018. It is the sixth most common cancer worldwide, but the third leading cause of cancer-related deaths. Another study found that gastric carcinoma is one of the most common cancers and the second leading cause of cancer-related death in the world. Despite decline morbidity and mortality, the burden remains high (McLean and EI-Omar, 2014).

Colon cancer is a type of cancer that begins within the large intestine (colon). The colon is the final part of the digestive tract. Colon cancer is sometimes referred to as colorectal cancer, which is a term that combines colon cancer and rectal cancer, which begins in the rectum. In the United States, Colorectal cancer is the second leading cause of cancer death among men and women combined. Around $25 \%$ of people diagnosed with colorectal cancer have a family history (fightcolorectalcancer.org/prevent-it/facts-about-colorectal-cancer/). The risk of cancer increases significantly with age and many cancers occur more commonly in developed countries. Rates are increasing as more people live to an old age and as lifestyle changes are occurring in the developing world (Jemal et al., 2011).

Cancer is a major reason behind death worldwide including Bangladesh. The incidence of various cancers is increasing day by day. The frequency of various forms of cancer occurring in Bangladesh is not similar to that of other worldwide population groups. To the best of our knowledge, no study has been conducted in Bangladesh to investigate the survival probabilities of stomach and colon cancer patients according to sex, age, and body mass index. Therefore, we aim to differentiate the survival probabilities of the colon and stomach cancer patients at the national level according to sex, age, and body mass index. This study reveals that female patients, patients older than 45 years and overweight patients have less median survival time than male, younger patients and healthy weight patients respectively.

\section{Materials and Methods}

\subsection{Ethics approval and consent to participate}

All procedures performed in studies involving human participants were in accordance with the ethical standards of the institutional and/or national research committee and with the 1964 Helsinki declaration and its later amendments or comparable ethical standards. The study was approved by the local ethics committee (University Research Center, Shahjalal University of Science and Technology, Sylhet, Bangladesh) and the ethical guidelines were strictly followed throughout the data collection. Written informed consent was obtained from all individual participants included in the study.

\subsection{Participants and data collection}

To differentiate the survival probabilities of the colon and stomach cancer patients according to sex, age and body mass index, initially a primary dataset was collected based on a questionnaire from eight divisional cities in Bangladesh using a convenient sampling. We found a total of 65 stomach and colon cancer patients during October 2018. Especially we collected the starting time of the diseases. After one year we communicated with them/relatives and then collected their status. We computed patients' survival time using the starting time of the disease and their death time. Remaining patients are considered as censored as they are still alive. The study was approved by the University Research Center, Shahjalal University of Science and Technology (SUST), Sylhet, Bangladesh and the ethical guidelines were strictly followed throughout the data collection. We informed participants about the purpose of the study before the data collection. 


\subsection{Statistical analyses}

Kaplan-Meier product limit estimate (Kishore and Khanna, 2010; Kleinbaum and Klein, 2011) with Log-rank test were used to estimate the survival probabilities of stomach and colon cancer patients. We used SPSS 22.0 for data analysis.

\section{Results}

In this study we tried to find out survival time of stomach and colon cancer patients as an outcome and then compare the survival probabilities respect to gender, age and body mass index. As sample size is very small therefore, we considered age and body mass index only two categories. Initially we tried to run the Cox model with all explanatory variables (gender, age, and BMI) with their interactions but none is found significant therefore we strict our analysis only to estimate K-M survival probabilities and Log rank test. The study included 46 stomach cancer (71\%) and 19 colon cancer patients (29\%). Among the 65 patients, 41 patients $(63 \%)$ are male and 24 patients (37\%) are female. After one year we found 20 patients (30.8\%) have died where 15 stomach patients and 5 colon patients. We observed the minimum and maximum age of the respondents was 11 and 80 years respectively. Almost two thirds (63.1\%) were male respondents. Among male patients, most of them are from the Sylhet division followed by Rangpur, Chattogram, and Dhaka, and among female patients, most of them are from Sylhet and Rajshahi followed by Chattogram division (Figure 1).

\subsection{Kaplan-Meier product limit estimates}

In order to compare the survival probabilities of male and female patients we plotted the K-M survival curve (Figure 2), it is shown that the survival probabilities of male patients are comparatively higher than female patients. From Table 1, it is also observed that median survival time is higher for male patients than female patients.

In order to compare the survival probabilities of different age groups, we divided the patients into two groups (less than or equal to 45 years and more than 45 years). We plotted K-M survival curves (Figure 3), it is shown that the survival probabilities of young groups (less than or equal to 45 years) are slightly higher than comparatively old age patients. We also observed in Table 2 that the median survival time is higher ( 31 months) in the younger group than the older group (21 months).

In order to compare the survival probabilities based on body mass index, we divided the patients into two groups (healthy weight and overweight (BMI $\geq 25$ ). We plotted K-M survival curves (Figure 4), it is shown that survival probabilities of the healthy weight group are higher than the overweight group. We also observed in Table 3 that median survival time is higher (30 months) in healthy weight group than overweight group (23 months).

Table 1. Medians for survival time according to gender.

\begin{tabular}{|l|l|l|l|l|}
\hline & \multirow{2}{*}{ Estimate } & \multirow{2}{*}{ Std. error } & \multicolumn{2}{|c|}{ 95\% Confidence interval } \\
\cline { 5 - 6 } & & & Lower bound & Upper bound \\
\hline Male & 31.000 & 4.729 & 21.731 & 40.269 \\
\hline Female & 22.000 & 5.930 & 10.376 & 33.624 \\
\hline
\end{tabular}

Table 2. Medians for survival time according to categorical age.

\begin{tabular}{|l|l|l|l|l|}
\hline & Estimate & Std. error & \multicolumn{2}{|c|}{ 95\% Confidence interval } \\
\hline & & & Lower bound & Upper bound \\
\hline Less than or equal to 45 years & 31.000 & 3.708 & 23.733 & 38.267 \\
\hline Greater than to 45 years & 22.000 & 5.231 & 11.748 & 32.252 \\
\hline
\end{tabular}

Table 3. Medians for survival time according to body mass index.

\begin{tabular}{|l|l|l|l|l|}
\hline & Estimate & Std. error & \multicolumn{2}{|c|}{ 95\% Confidence interval } \\
\hline & & & Lower bound & Upper bound \\
\hline Within a healthy weight range & 30.000 & 6.971 & 16.338 & 43.662 \\
\hline Overweight & 23.000 & 13.880 & .000 & 50.206 \\
\hline
\end{tabular}




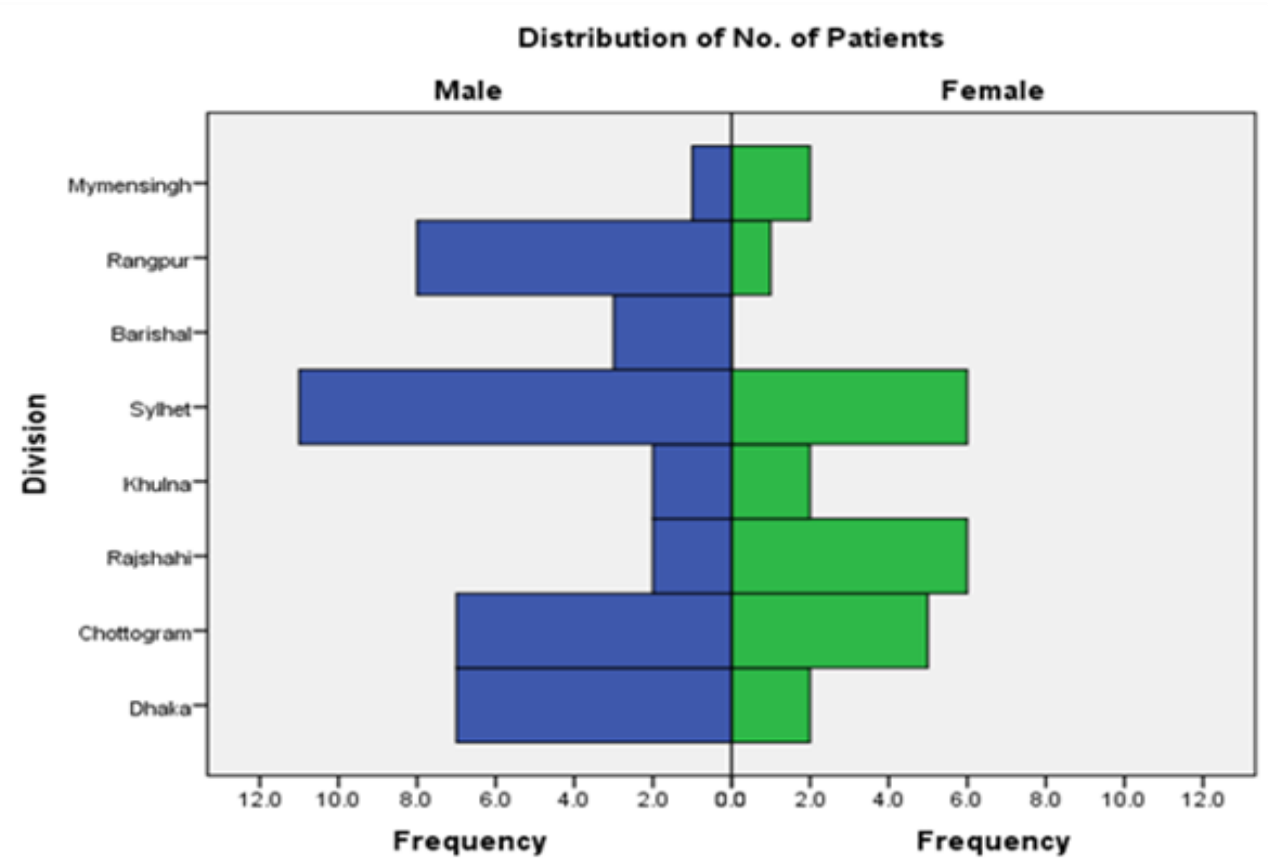

Figure 1. Distribution of the number of stomach and colon cancer patients by division and gender.

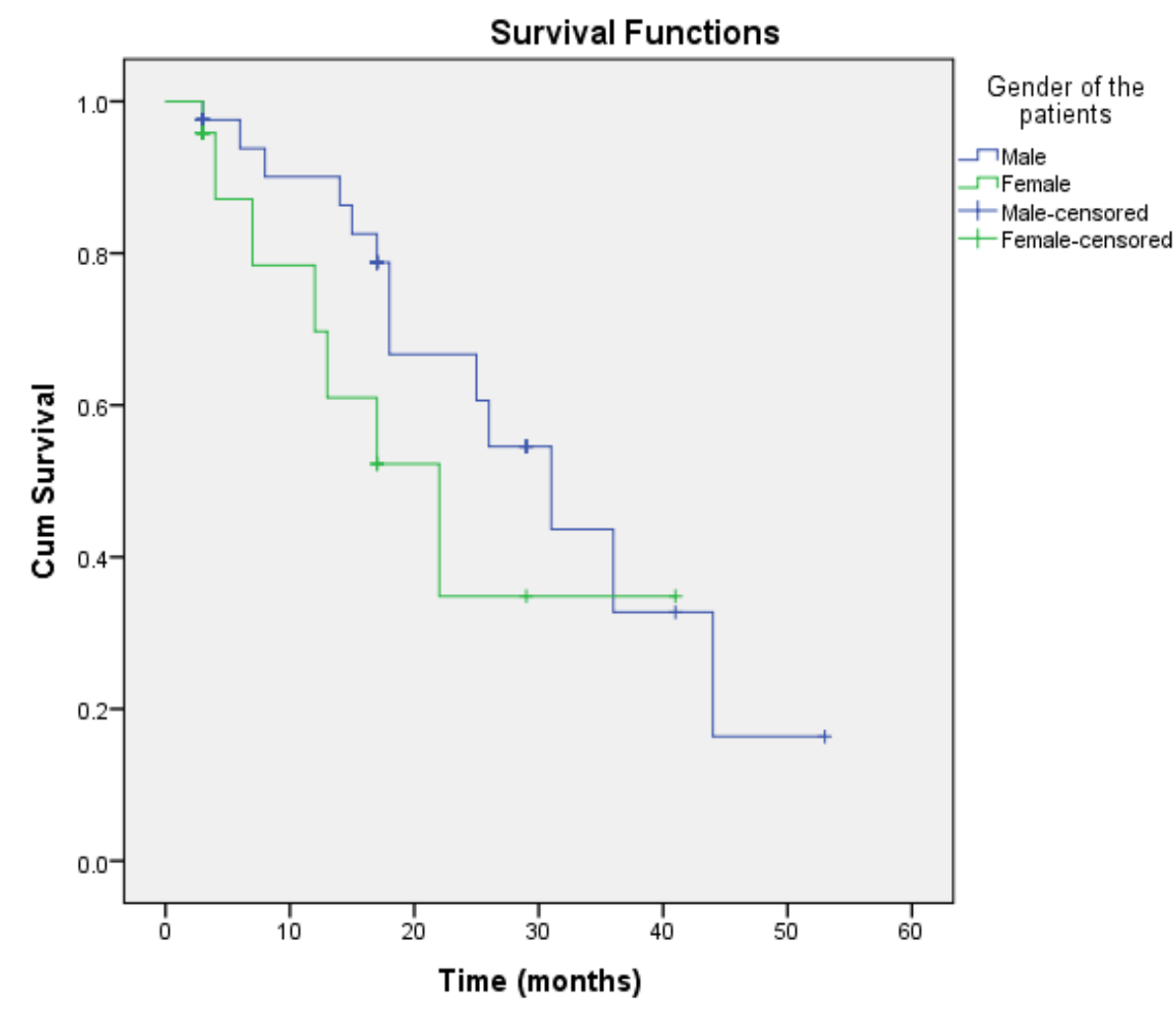

Figure 2. Plots of Kaplan-Meier product limit estimates of survival according to gender. 


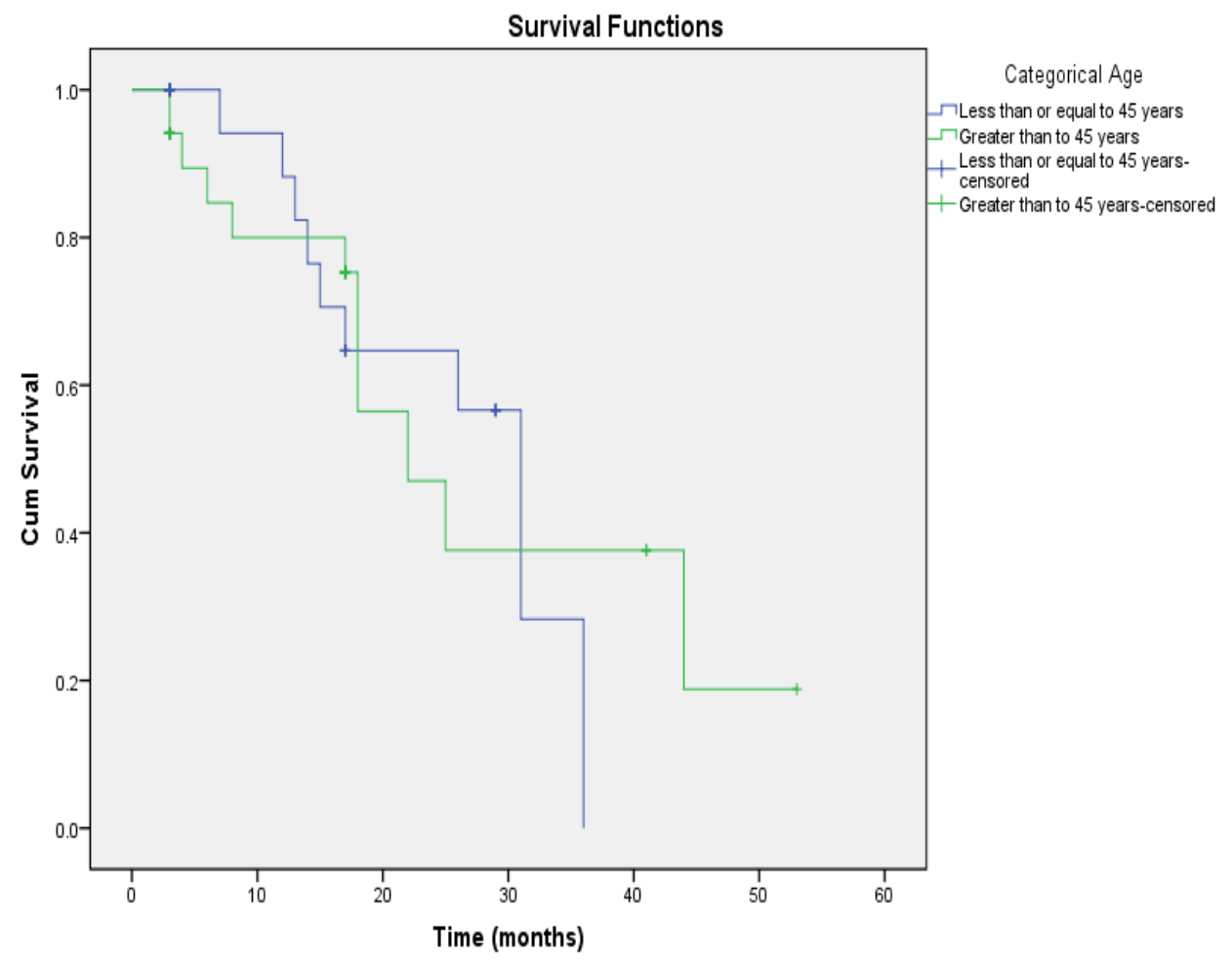

Figure 3. Plots of Kaplan-Meier product limit estimates of survival according to the age of the patients.

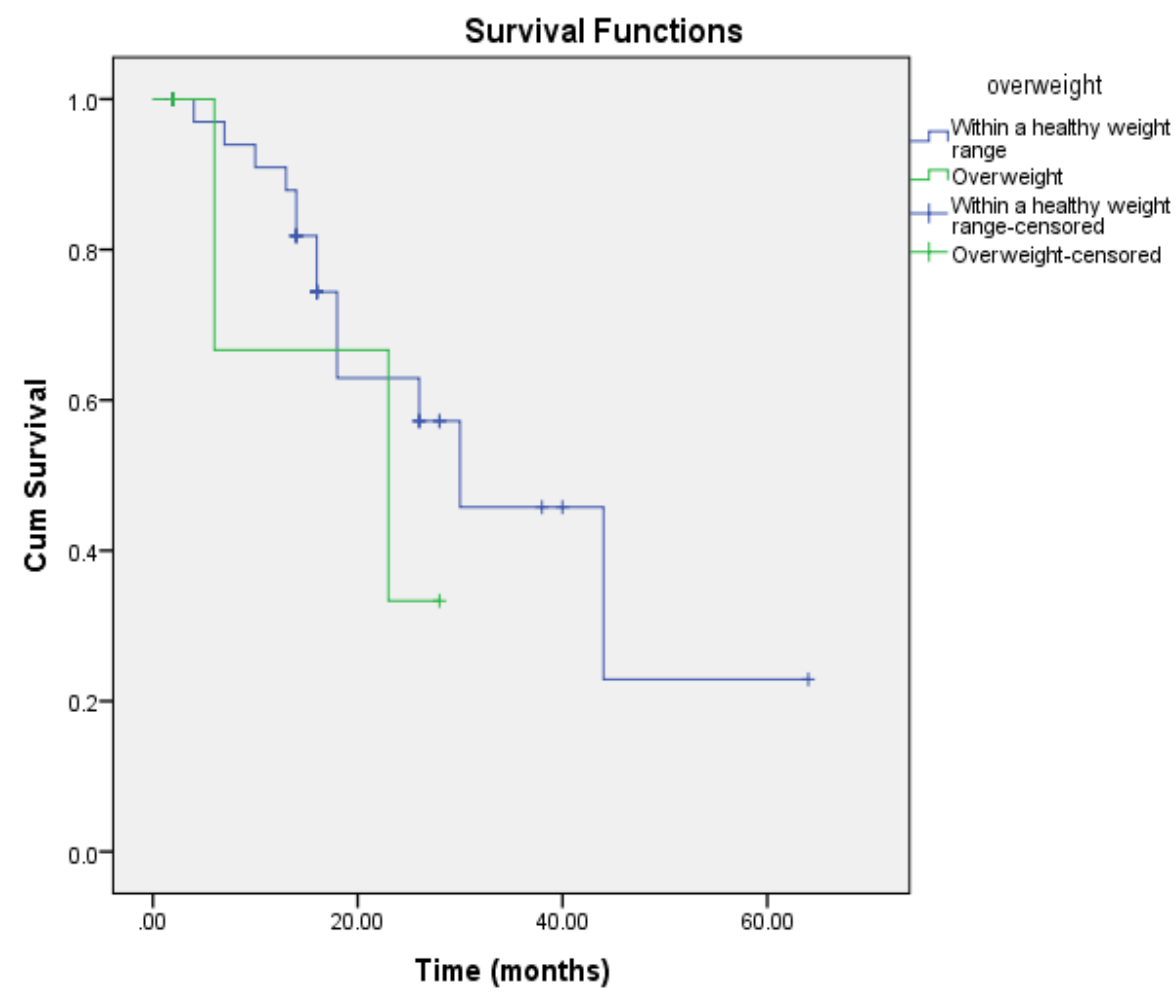

Figure 4. Plots of Kaplan-Meier product limit estimates of survival according to body mass index.

\section{Discussion}

Cancer affects most of us at some point in our lives. Cancer cases are expected to surge 57\% worldwide in the next 20 years, an imminent "human disaster" that will require a renewed focus on prevention to combat, according to the World Health Organization. The World Cancer Report, produced by the WHO's specialized cancer agency and released on World Cancer Day, predicts new cancer cases will rise from an estimated 14 
million annually in 2012 to 22 million within two decades. Over the same period, cancer deaths are predicted to rise from 8.2 million a year to 13 million. Low- and middle-income countries accounted for about half (51\%) of all cancers worldwide in 1975; this proportion increased to 55\% in 2007 and is projected to reach $61 \%$ by 2050 (Bray and Møller, 2006). Incidence and death rates from stomach cancer have steadily declined over the last 50 years, even though stomach cancer remains the fourth most common malignancy and is second only to lung cancer as the leading cause of cancer deaths (Parkin et al., 2002). Stomach cancer accounted for $>1$ million estimated cases and 800230 deaths in 2007 , with an estimated $60 \%$ of new cases occur in developing countries. Colorectal cancer was rather rare in 1950 but has become one of the predominant cancers in western countries, now accounting for approximately $10 \%$ of cancer-related mortality (Vasen et al., 2015). Although it is possible for cancer to strike at any age, most patients with invasive cancer are over 65 (Coleman and Rubinas, 2009). According to cancer researcher Robert A. Weinberg, "If we lived long enough, sooner or later we all would get cancer" (Johnson, 2010). Aging's effect on cancer is complicated by factors such as DNA damage and inflammation-promoting it and factors such as vascular aging and endocrine changes inhibiting it (De Magalhaes, 2013).

The results of Log-rank tests according to three cases (sex, age, and BMI) showed there is no significant difference between male vs female; older vs younger and healthy weight vs overweight of survival probabilities of cancer patients, however, observing the Figures 2, 3 and 4- we clearly see that there is a difference between the respective groups. Based on the exploratory data analysis we found male, younger and healthy patients' survival probabilities are comparatively higher than female, older and overweight patients respectively which is because of the well-known and established facts that women's and older peoples' immune system is not as stronger as males' and younger people. This study also suggests that overweight decreases the survival time which supports the previous studies as well.

\section{Conclusions}

Including all, we conclude that male, younger and healthy weight patients' survival probabilities are higher than female, older and overweight patients respectively. To improve the survival probabilities of cancer patients, government and non-government organizations need to increase their campaign about the causes and consequences of cancer for enhancing awareness levels on cancer. It is also important for people to take a balanced diet and exercise regularly to keep their healthy weight. Though, the Bangladesh government has been planning to set up cancer centers in eight medical colleges to reach out to these patients; however, it is urgently needed to create a data-house in each hospital so that doctors/researchers can get the exact picture of the cancer status in Bangladesh.

\section{Acknowledgements}

We would like to thank the SUST research center for the partial fund of this study. We also want to thank the authority and patients for their cordial support during the data collection.

\section{Conflict of interest}

None to declare.

\section{References}

World Health Organization, 2008. The Global Burden of Disease: 2004 Update. Geneva: World Health Organization.

Jemal A, F Bray, MM Center, J Ferlay, E Ward and D Forman, 2011. Global cancer statistics. CA Can. J. Clin., 61: 69-90.

Population fact sheets: Bangladesh, 2018. Retrieved from https:/gco.iarc.fr/today/data/factsheets/ populations/50-bangladesh-fact-sheets.pdf

Bray F, J Ferlay, I Soerjomataram, RL Siegel, LA Torre and A Jemal, 2018. Global Cancer Statistics 2018: GLOBOCAN Estimates of Incidence and Mortality Worldwide for 36 Cancers in 185 Countries. CA Can. J. Clin., 68: 394-424.

Ferlay J, F Bray, P Pisani and DM Parkin, 2004. GLOBOCAN 2002 cancer incidence, mortality and prevalence worldwide. IARC Cancer Base No. 5, version 2.0. Lyon: IARC Press; 2004.

Janssen-Heijnen ML, S Houterman, VE Lemmens, MW Louwman, HA Maas and JW Coebergh, 2005. Prognostic impact of increasing age and co-morbidity in cancer patients: a population-based approach. Crit. Rev. Oncol. Hemat., 55: 231-240. 
Sant M, R Capocaccia, AVerdecchia, G Gatta, A Micheli and A Mariotto, 1995. Comparisons of colon-cancer survival among European countries: the eurocare study. Int. J. Can., 63: 43-48.

Capocaccia R, R De Angelis, L Frova, G Gatta, M Sant, A Micheli, F Berrino, E Conti, L Gafà, L Roncucci and A Verdecchia, 1997. Estimation and projections of colorectal cancer trends in Italy. Int. J. Epidemiol., 26: 924-932.

Li M, JY Li, AL Zhao and J Gu, 2007. Colorectal cancer or colon and rectal cancer? Clinicopathological comparison between colonic and rectal carcinomas. Oncology, 73: 52-57.

Cheng X, VW Chen, B Steele, B Ruiz, J Fulton, L Liu, SE Carozza and R Greenlee, 2001. Subsite-specific incidence rate and stage of disease in colorectal cancer by race, gender, and age group in the United States, 1992-1997. Cancer, 92: 2547-2554.

Ji BT, SS Devesa, WH Chow, F Jin and YT Gao, 1998. Colorectal cancer incidence trends by subsite in urban Shanghai, 1972- 1994. Cancer Epidemiol. Biomarkers Prev., 7: 661-666.

Svensson E, T Grotmol, G Hoff, F Langmark, J Norstein and S Tretli, 2002. Trends in colorectal cancer incidence in Norway by gender and anatomic site: an age-periodcohort analysis. Eur. J. Cancer Prev, 11: 489- 95.

Wynder EL and T Shigematsu, 1967. Environmental factors of cancer of the colon and rectum. Cancer, 20: 1520-1561.

Lew EA and L Garfinkel, 1979. Variations in mortality by weight among 750,000 men and women. J. Chron. Dis., 32: 563-576.

Phillips RL and DA Snowdon, 1985. Dietary relationships with fatal colorectal cancer among Seventh Day Adventists. J. Nar. Cancer Insr., 74: 307-317.

Wu AH, A Paganinifhill, RK Ross and BE Henderson, 1987. Alcohol, physical activity and other risk factors for colorectal cancer: a prospective study. Brit. J. Cancer, 55: 687-694.

Graham S, J Marshall, B Haughey, A Mittelman, M Swanson, M Zielezny, T Byers, G Wilkinson and D West, 1988. Dietary epidemiology of cancer of the colon in Western New York. Amer. J. Epidemiol., 128: 490503.

Kune GA, S Kune and LF Watson, 1990. Body weight and physical activity as predictors of colorectal cancer risk. Nutr. Cancer, 13: 9-17.

Hou L, B-T Ji, A Blair, Q Dai, Y-T Gao, JD Potter and W-H Chow, 2006. Body mass index and colon cancer risk in Chinese people: Menopause as an effect modifier. Eur. J. Can., 42: 84-90.

Hayne D, RSD Brown, M McCormack, MJ Quinn, HA Payne, P Babb, 2001. Current trends in colorectal cancer: site, incidence, mortality and survival in England and Wales. Clinical Oncology, 13: 448- 452.

Payne S, 2007. Not an equal opportunity disease - a sex and gender-based review of colorectal cancer in men and women: Part I. JMHG, 4: 131-139.

Van Cutsem E, X Sagaert, B Topal, K Haustermans, H Prenen, 2016. Gastric Cancer. The Lancet, 388: 26542664.

Karimi P, F Islami, S Anandasabapathy, ND Freedman and F Kamangar, 2014. Gastric Cancer; descriptive epidemiology, risk factors, screening, and prevention. Cancer Epidemiol Biomarkers Prev., 23: 700 - 713.

McLean MH and EM EI - Omar, 2014. Genetics of gastric cancer. Nat. Rev. Gastroenteror. Hepatol., 11: 664 674.

Jemal A, F Bray, MM Center, J Ferlay, E Ward and D Forman, 2011. "Global cancer statistics". Ca. 61: 69-90.

Kishore J, M Goel and P Khanna, 2010. Understanding survival analysis: Kaplan-Meier estimate. Int. J. Ayur. Res., 1: 274.

Kleinbaum DG and M Klein, 2011. Kaplan-Meier Survival Curves and the Log-Rank Test. Survival Analysis, 55-96.

Bray F and B Møller, 2006. Predicting the future burden of cancer. Nat. Rev. Cancer., 6: 63-74.

Parkin DM, F Bray, J Ferlay and P Pisani, 2005. Global cancer statistics, 2002. CA Cancer J. Clin., 55: 74-108.

Vasen HFA, I Tomlinson and A Castells, 2015. Clinical management of hereditary colorectal cancer syndromes. Nat. Rev. Gastroenterol. Hepatol., 12: 88 -97.

Coleman WB and TC Rubinas, 2009. In Tsongalis GJ, Coleman WL (eds.). MolecularPathology: The Molecular Basis of Human Disease. Amsterdam: Elsevier Academic Press. p. 66.

Johnson G, 2010. "Unearthing Prehistoric Tumors, and Debate". The New York Times. Archived from the original on 24 June 2017.

de Magalhães JP, 2013. "How ageing processes influence cancer". Nature Reviews. Cancer, 13: 357-365. 